\title{
Studies of a family with incontinentia pigmenti variably expressed in both sexes
}

\author{
THADDEUS W KURCZYNSKI, JEFFREY S BERNS, AND WALTER E JOHNSON
}

\begin{abstract}
From the Departments of Pediatrics and Neurology and the Genetics Center, Case Western Reserve University and Rainbow Babies and Childrens Hospital, Cleveland, Ohio 44106, USA.
\end{abstract}

SUMMARY A family is described in which incontinentia pigmenti (IP) is variably expressed in both sexes, compatible with either autosomal dominant or $\mathrm{X}$ linked dominant inheritance. This is the first reported instance of an affected male with a positive family history. Immunological studies of the proband showed no significant alteration of immune function. Cytogenetic investigations of the proband and her affected brother and mother revealed no unusual propensity for chromosome breaks or gaps and no enhancement of sister chromatid exchanges.

Incontinentia pigmenti (Bloch-Sulzberger syndrome) is a familial multisystem disorder manifested by characteristic dermatological lesions associated with variable abnormalities of the teeth, hair, nails, eyes, blood, and central nervous system. ${ }^{1}$ The skin lesions are usually present at birth, or appear shortly thereafter, and are initially characterised by an erythematous eruption with linear vesiculation and an associated leucocytosis and eosinophilia followed by verrucous growths which eventually resolve spontaneously leaving areas of atrophy or depigmentation or both. ${ }^{2}$

The majority of patients have dental anomalies which may involve the deciduous or permanent dentition. The scalp may show variable alopecia. Nail dystrophy occurs in a minority of cases. Ocular abnormalities occur in about a third of patients and may produce serious visual deficits. Potentially the most serious involvement is in the central nervous system which is affected in approximately one-third of patients and may result in seizures, mental retardation, or spastic paralysis.

Various other structural anomalies may occur in incontinentia pigmenti but do not appear to be consistent or characteristic of this disorder. A history of frequent infections has been noted by various authors who have suggested the possibility of an immune dysfunction. ${ }^{3-5}$

The genetic basis for IP has not been resolved. The disorder has been reported in various ethnic groups and geographical areas but is almost exclusively confined to females. A positive family history

Received for publication 17 March 1982. is obtained in over half of the female patients. ${ }^{1}$ Affected males, however, are extremely rare and none has been identified with a family history. ${ }^{1}$ Pfeiffer $^{6}$ suggested autosomal dominant inheritance with expression only in females while Lenz ${ }^{7} 8$ postulated an $\mathrm{X}$ linked dominant gene lethal in males.

Chromosome studies in some patients have shown an increased frequency of chromosome breakage although no systematic rearrangement has been described. ${ }^{9-13}$

The family reported here is the first described in which genetic transmission of IP resulted in an affected male and female. There was no suggestion of lethality or greater severity in the affected male. Chromosome studies of the leucocytes from three affected family members, as well as immunological investigations of the proband, were carried out and provide further data on the expression of this genetic disease.

\section{Case reports}

The proband was a 9-month-old female infant who was referred for genetic evaluation. She was the product of uncomplicated term pregnancy, labour, and delivery with a birth weight of $4026 \mathrm{~g}$. Unusual vesicular lesions arranged in linear patterns were noted at birth mostly on the lower extremities and trunk and to a lesser degree on the upper extremities. These lesions gradually developed a brownish pigmentation and macular appearance although a few displayed a verrucuous pattern. At the age of 10 weeks she was admitted to hospital with acute 


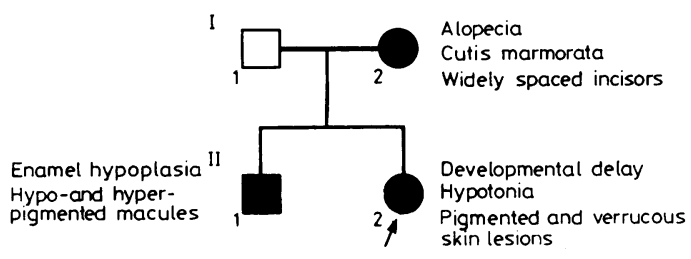

FIG 1 Pedigree showing variable manifestations of incontinentia pigmenti in both sexes.

swelling of the left wrist suspected of resulting from osteomyelitis. No lesions were found on $x$-ray and the swelling cleared with antibiotic therapy. There were multiple episodes of otitis media but there was no history of ear drainage, pneumonia, or other serious infections. Developmental landmarks were normal.

A pedigree is shown in fig 1 . An older male sib was 3 years old and in good health except for a history of repeated dental evaluations for enamel hypoplasia particularly affecting the incisor teeth. He had no history of similar skin lesions as an infant and no other history of medical problems. The mother was 25 years old and except for allergies was in good health. She had had no miscarriages or stillbirths. As an infant she had blister-like skin lesions similar to her daughter's and these eventually resolved. The skin over her lower extremities, however, was always mottled. In addition, her incisors were widely spaced and she had a persistent patch of alopecia. The mother was the second oldest of a sibship of 13 with five males and eight females. There was, in addition, one female stillborn and one female infant who died at 3 weeks with bowel eventration. Two of the mother's sisters, aged 20 and 12, and a 9-year-old brother also had mottling of the skin. Another 10-year-old brother and the mother's 46year-old mother had widely spaced teeth similar to the mother's but no history of skin lesions, alopecia, or other medical problems.

Examination of the proband showed a head circumference of $45 \mathrm{~cm}$ (75th centile), weight $8.75 \mathrm{~kg}$ (50th to 75 th centile), and length $69.5 \mathrm{~cm}$ (50th centile). There were brownish macular-papular lesions distributed in whorled or linear patterns over the scalp, trunk, and extremities, particularly in the lower extremities, on the left more than the right. In addition, there were brownish verrucous lesions over the second left toe, the left index finger, and the right thumb. Figs 2 and 3 illustrate the skin lesions. The palate was high arched and there were no palpable teeth except for one minimally erupted pegshaped incisor. The general examination was otherwise unremarkable. Neurological examination was significant only for a mild generalised flexion of the trunk when sitting upright, and horizontal suspension demonstrated decreased ability to extend the neck and hips. The deep tendon reflexes were normal.

Examination of the proband's 3-year-old brother was positive only for enamel hypoplasia involving the upper and lower incisors, the presence of scattered linear and more rounded hypopigmented lesions on the thorax and extremities, and a hyperpigmented patch over the right abdomen.

The mother's examination was significant for a patch of alopecia over the left posterior parietal area, widely spaced upper and lower incisors with normal enamel, and prominent cutis marmorata over the lower extremities. The mother's family members were not available for examination.

Laboratory studies of the proband included a normal CBC with no eosinophilia. The following all
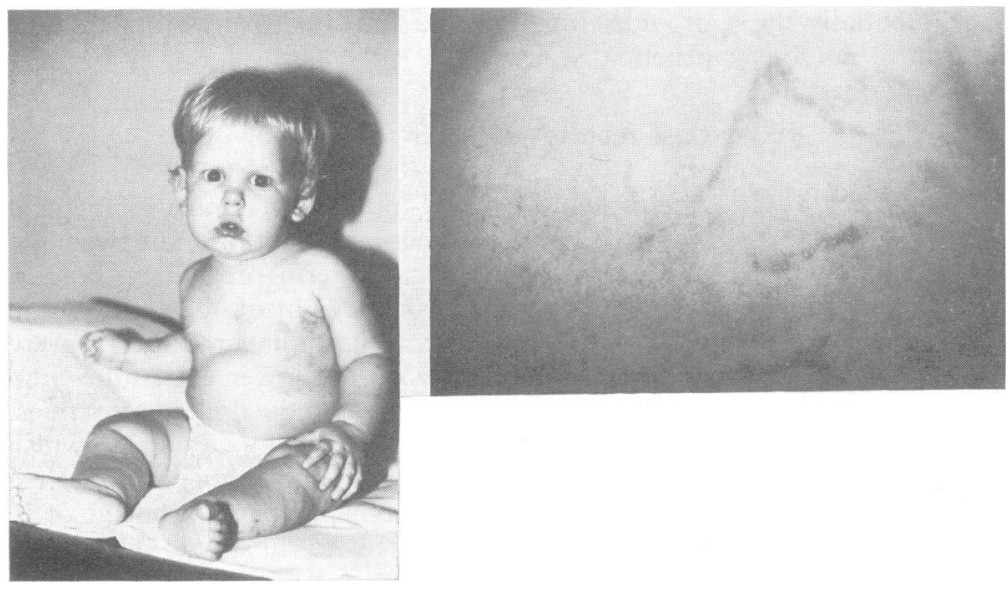

FIG 2 Pigmented and verrucous lesions of incontinentia pigmenti in the proband. 


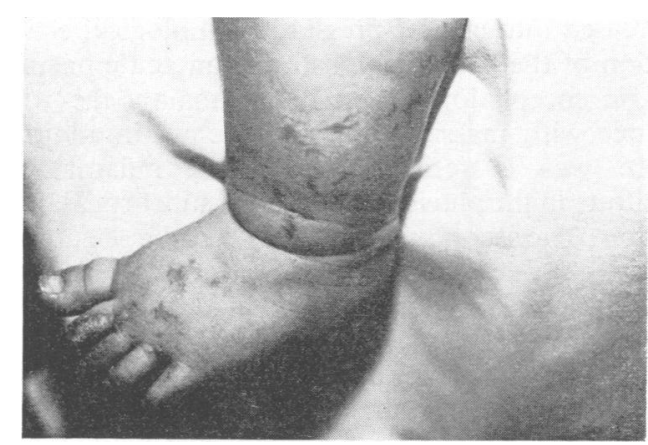

FIG 3 Pigmented and verrucous lesions on the left leg and foot in the proband.

gave normal results: urine analysis, serum electrolytes, BUN, glucose, $\mathrm{Ca}, \mathrm{P}$, alkaline phosphatase, creatinine, SGPT, LDH, CK, total bilirubin, total protein, albumin, immunoglobulin levels, $T$ and $B$ lymphocytes, and neutrophil chemotaxis. Urine metabolic screening demonstrated no abnormal amino-aciduria.

An EEG was normal. A CT scan of the head showed generalised ventricular dilation and prominence of the basal cisterns. An EMG and nerve conduction studies were unremarkable. A skin biopsy demonstrated some increased keratin, modest vacuolisation in the basal epidermal cells, and pigment located in dermal melanophages.

Chromosome studies were performed on peripheral blood leucocytes from the proband, her brother, and the mother. No abnormality was found in trypsin $G$ banded preparations from all three subjects. No chromosome breaks or gaps were seen in examination of 60 cells from the proband and 20 cells from the mother. A single chromosome break was seen in 60 cells from the brother. Sister chromatid exchanges were prepared and scored by the method of Goto et al..$^{14}$ A average of 4.58 exchanges per cell were found for the proband and 5.55 for her brother, which are both within the normal range for our laboratory.

\section{Discussion}

The family reported here illustrates the marked variability in expression of the gene for incontinentia pigmenti. The proband by history and examination manifested the typical skin lesions and in addition had central nervous system involvement demonstrated by the ventricular enlargement and hypotonia. The presence of only one minimally erupted pegshaped incisor and the lack of specific palpable teeth at 9 months of age may indicate dental abnormalities, but the young age of the proband makes these findings inconclusive. The proband's brother, however, had not only definite thinning of tooth enamel but various hypopigmented macules and a hyperpigmented patch. The hypopigmented streaks may in some patients represent the eventual sole dermatological manifestation of the disease. Wiley and Frias ${ }^{2}$ emphasised the value of these lesions in the diagnosis of asymptomatic relatives or when other signs are equivocal. The mother was the only member of a sibship of 15 to have skin lesions in early infancy similar to her affected daughter. At present, her only skin manifestations are a circumscribed area of scalp alopecia and a prominent cutis marmorata of the lower extremities. The mother reported similar changes in the skin of two sisters, aged 20 years and 12 years, and a 9-year-old brother. This brother, a 10-year-old brother, and the mother's 46-year-old mother also have gaps between the teeth but no other history of abnormalities of the skin, hair, eyes, or nervous system.

The presence of cutis marmorata or livedo reticularis in patients with incontinentia pigmenti has also been described in a family reported by Reed $e t$ al. ${ }^{15}$ Although the later skin manifestations of IP commonly assume a reticular pattern, the nature of the lesions and other associated abnormalities usually serve to distinguish IP from benign cutis marmorata or livedo reticularis. In a few patients, however, such as the mother of the proband and possibly some of her sibs, the cutis marmorata may represent another manifestation of the IP gene. Unfortunately, the mother's sibs could not be examined and the limited information available suggests, but is insufficient for, the diagnosis of IP.

The occurrence of a family with incontinentia pigmenti affecting both sexes has not been noted previously. Carney, ${ }^{1}$ in a review of published reports of incontinentia pigmenti, identified 653 cases but only 16 were males. The few living males reported seemed to have the same variable manifestations as their female counterparts, and there was no suggestion of greater severity in the affected male. All reported male cases to date have been sporadic, presumably arising as a consequence of new mutations. The presence of an affected mother, son, and daughter is more in favour of the disorder being the consequence of an autosomal dominant gene than an $\mathrm{X}$ linked dominant gene lethal in males. $\mathrm{X}$ linked inheritance, however, cannot be excluded in this family. One male affected with IP was found to be $\mathrm{XXY}$ but not all have been karyotyped. ${ }^{16}$ It is also possible that there are in fact two different genes giving rise to IP, one autosomal with predominant limitation of expression to females, and the other $\mathrm{X}$ linked and lethal in males. Hopefully, as additional families with affected males are studied the mode of 
inheritance and possible genetic heterogeneity will be clarified.

Cytogenetic investigations of the three affected subjects in the family reported here disclosed no evidence of either abnormal chromosome breakage or increased sister chromatid exchange. de Grouchy et $a l^{9}$ were the first to report increased chromosome breakage in the peripheral blood leucocytes of a female with IP. Other cytogenetic studies of IP families have been reported by Cantu et al,$^{10}$ Iancu et $a l,{ }^{11}$ Kelly et $a l^{12}$ and Vissian et al. ${ }^{13}$ These investigators found increased chromosome breakage in some, but not all, patients as well as in some unaffected relatives. These data and the negative cytogenetic findings in our family raise questions about the significance and specificity of increased chromosome breakage reported in some families with incontinentia pigmenti. The lack of any chromosomal abnormalities in the affected male of our family suggests that if there is a specific relationship of chromosome breaks in incontinentia pigmenti, it is not more pronounced in the rare affected male. The lack of any suggestion of increased incidence of malignancies in incontinentia pigmenti, a known phenomenon with established chromosome instability syndromes such as ataxia telangiectasia, Bloom syndrome, Fanconi anaemia, and xeroderma pigmentosum, also raises doubts about the specificity of the findings. Systematic cytogenetic evaluations of more families with incontinentia pigmenti will be needed to clarify the relationship and significance of chromosome breakage in incontinentia pigmenti.

Reports of immune dysfunction in patients with incontinentia pigmenti have been limited and inconsistent. Our proband showed no immunological abnormalities. It is, therefore, more likely that the rare immunological abnormalities are unrelated to incontinentia pigmenti.

The possibility that a specific inflammatory process may play a role in the pathogenesis of incontinentia pigmenti is raised by a number of observations. The earliest histological changes in the epidermis include an inflammatory infiltrate with a predominance of eosinophils, and occasionally eosinophilia. ${ }^{17}$ Hauw et al, ${ }^{18}$ in a neuropathological study of a 3-month-old infant girl, found a diffuse inflammatory process involving the pia-arachnoid and brain parenchyma. They noted perivascular cuffs of lymphocytes, histiocytes, and eosinophils as well as mononuclear nodules. Moreover there was ulegyria and cavitation apparently secondary to hypoxia or ischaemia or both. Siemens et al ${ }^{19}$ described three sisters with incontinentia pigmenti who manifested inflammatory changes in the nervous system. The oldest girl developed post-vaccinial encephalitis which resolved, but 4 months later she deteriorated with seizures and increased intracranial pressure. Pathological examination of the brain demonstrated an acute haemorrhagic encephalopathy involving mainly the white matter with massive cerebral oedema. In addition, there was a residual perivenous inflammatory infiltrate in the anterior and central subcortical white matter. The second sister had an afebrile seizure at 8 months, 3 weeks after her first injection of diptheria and tetanus toxoid. At 2 years of age she developed a seizure, lethargy, and vomiting which was preceded by an upper respiratory infection. An LP showed nine lymphocytes and a strongly positive Pandy. A CT scan demonstrated a hypodense white matter lesion on the left. She improved but was left with residual spastic paraplegia greater on the left. The youngest sister suffered from an afebrile convulsion at the age of 5 months with a normal CSF. At 2 years of age she died after an acute illness manifested by fever, vomiting, seizures, and coma. Neuropathological findings were similar to those of the oldest sister, showing an acute haemorrhagic and residual perivenous encephalitis. Siemens et $a l^{19}$ postulated an 'allergic' basis for the neurological manifestations of incontenentia pigmenti based on these three cases. Additional data are needed to determine whether these neuropathological observations represent incidental coexisting disease or reflect a specific type of inflammatory process in incontinentia pigmenti.

\section{References}

1 Carney RG Jr. Incontinentia pigmenti: a world statistical analysis. Arch Dermatol 1976;112:535-42.

2 Wiley HE, Frias JL. Depigmented lesions in incontinentia pigmenti. Am J Dis Child 1974;128:546-7.

3 Honig PJ, Miller ME. Incontinentia pigmenti-a possible immunologic disorder. J Pediatr 1972;80:334-6.

4 Dahl MV, Matula G, Leonards R, Tuffanelli DL. Incontinentia pigmenti and defective neutrophil chemotaxis. Arch Dermatol 1975;111:1603-5.

5 Jessen RT, Van Epps DE, Goodwin JS, Bowerman J. Incontinentia pigmenti: evidence for both neutrophil and lymphocyte dysfunctions. Arch Dermatol 1978;114:11826.

6 Pfeiffer RA. Zur Frage der Vererbung der Incontinentia pigmenti Block-Siemens. $Z$ Mensche Vererb Konstitutional 1960;35:469-93.

7 Lenz W. Zur Genetik der Incontinentia Pigmenti. Ann Paediatr 1961 ;196:149-65.

8 Lenz W. Half chromatid mutations may explain incontinentia in males. Am J Hum Genet 1975;27:690-1.

9 de Grouchy J, Bonnetti J, Brussieux J, Roidot M, Begin P. Cassures chromosomiques dans l'incontinentia pigmenti. Ann Genet (Paris) 1972;15:61-5.

10 Cantu JM, del Castillo V, Jimenez M, Ruiz-Barquin E. Chromosomal instability in incontinenia pigmenti. Ann Genet (Paris) 1973;16:117-9.

11 Iancu T, Komlos L, Shabtay F, Elian E, Halbrecht I, Book JA. Incontinentia pigmenti. Clin Genet 1975;7: 103-10. 
12 Kelly TE, Rary JM, Young L. Incontinentia pigmenti : a chromosomal breakage syndrome. J Hered 1976;67: 171-2.

13 Vissian L, Emerit I, Levy A, Vailland JC. Incontinentia pigmenti, étude chromosomique d'une famille. Ann Dermatol Venereol 1978;105:119-21.

14 Goto K, Maeda S, Kano Y, Sugiyama T. Factors involved in differential Giemsa staining of sister chromatids. Chromosoma 1975;66:351-9.

15 Reed WB, Carter O, Cohen TM. Incontinentia pigmenti. Dermatologica 1967;134:243-50.

16 Kunze J, Frenzel UH, Huettig E, Grosse FR, Wiedemann HR. Kleinfelter's syndrome and incontinentia pigmenti Bloch-Sulzberger. Hum Genet 1977;35:237-40.

17 Carney RG. Incontinentia pigmenti: a report of five cases and review of the literature. Arch Dermatol 1951;64: 126-35.

18 Hauw JJ, Perie G, Bonnette J, Escourolle R. Les lesions cerebrales de l'incontinentia pigmenti. Acta Neuropathol (Berl) 1977;38:159-62.

19 Siemens H, Schneider H, Dening D, Hanefeld F. Encephalitis in two members of a family with incontinentia pigmenti (Bloch-Sulzberger syndrome). Eur $J$ Pediatr $1978 ; 129$ :103-15.

Requests for reprints to Dr T W Kurczynski, Department of Pediatrics, Division of Genetics, Medical College of Ohio, CS 10008, Toledo, Ohio 43699, USA. 